\title{
La démocratie et ses limites
}

Democracy and its Limits

民主與其侷限

\section{Emmanuel Terray}

\section{(2) OpenEdition}

Journals

Édition électronique

URL : http://journals.openedition.org/extremeorient/151

DOI : 10.4000/extremeorient.151

ISSN : 2108-7105

Éditeur

Presses universitaires de Vincennes

Édition imprimée

Date de publication : 1 octobre 2009

Pagination : 179-187

ISBN : 978-2-84292-237-5

ISSN : 0754-5010

Référence électronique

Emmanuel Terray, "La démocratie et ses limites », Extrême-Orient Extrême-Occident [En ligne],

31 | 2009, mis en ligne le 01 octobre 2012, consulté le 02 mai 2019. URL : http://

journals.openedition.org/extremeorient/151 ; DOI : 10.4000/extremeorient.151

(C) PUV 


\title{
La démocratie et ses limites
}

\author{
Emmanuel Terray
}

L'ensemble présenté par ce numéro d'Extrême-Orient Extrême-Occident est d'une richesse telle que le lecteur sollicité de réagir est bien obligé de choisir. On aimerait par exemple revenir sur la thèse défendue par Qin Hui selon laquelle, sous une apparente hégémonie confucéenne, c'est en réalité l'interaction entre le légisme et le taoïsme qui a constitué le courant dominant de l'histoire impériale, et peut-être de celle qui a suivi. On s'attarderait volontiers aussi sur les hypothèses de Gan Yang quant aux liens qui, durant la seconde moitié du Xx siècle, ont pu s'établir entre l'ère maoïste et l'ère des réformes: c'est l'hostilité déclarée de Mao à la planification économique centralisée selon le modèle soviétique qui aurait créé «les bases fondamentales pour la mise en œuvre ultérieure de la réforme économique de Deng Xiaoping ». Sur ces deux points, entre autres, on voudrait en apprendre beaucoup plus. Pourtant faute de place et surtout de compétence, je les laisserai de côté, et je m'en tiendrai à une seule question: celle de la démocratie et de ses limites.

Je partirai d'une constatation indiscutable: la démocratie européenne ou américaine - disons, pour faire bref, l'ensemble formé par les institutions représentatives et «l'État de droit»- est assurément un produit de la culture et de l'histoire occidentales ; plus précisément, elle est l'héritage que nous ont laissé les Lumières du XVIII ${ }^{e}$ siècle. Même si, du fait des principes et des valeurs dont elle se réclame, elle revendique le statut de modèle universel, exemplaire partout et pour tous, cette universalité n'est pas de soi évidente; elle doit, si faire se peut, être démontrée, de telle sorte qu'elle s'impose à tout être raisonnable. Il est donc naturel et juste que la démocratie à l'occidentale soit soumise à l'examen et au jugement des autres cultures: à elles de vérifier les prétentions émises et les titres produits. Telle est la tâche à laquelle s'attellent - chacun à sa manière - les six auteurs retenus par la revue; leur entreprise est non seulement légitime, mais nécessaire et par conséquent bienvenue. 
Or un trait commun apparaît aussitôt dans les six textes ici réunis : aucun d'eux ne récuse la démocratie occidentale du simple et unique fait qu'elle serait occidentale, qu'elle représenterait pour la société et la culture chinoises un apport étranger. Même ceux qui la critiquent le plus durement, et qui concluent le plus fermement à son inadaptation aux réalités chinoises, lui opposent des arguments de fond, touchant «the heart of the matter»; ils mettent en lumière des défauts ou des limites qui la caractérisent en tant que telle, dans son contenu propre, indépendamment de son origine historique. Autrement dit, nous sommes à mille lieues d'un débat en termes «culturalistes» ou encore de réquisitoires inspirés par le seul sentiment nationaliste, et nous ne pouvons que nous en féliciter.

La meilleure preuve en est que la plupart des objections ici soulevées trouvent un écho dans les controverses qui, à l'intérieur même de l'histoire intellectuelle de l'Occident, se sont poursuivies autour de la notion de démocratie. Qin Hui a parfaitement raison de souligner que la démocratie occidentale présente des visages variés: entre sa version antique et sa version moderne, entre les systèmes qui s'inspirent de Locke et ceux qui se réclament de Rousseau, subsistent des différences profondes, qui ont été l'objet de discussions intenses au sein du «camp démocrate». Au surplus, à l'extérieur de celui-ci, le principe même de la démocratie a été contesté par des penseurs aussi divers et considérables que Platon, Burke, Joseph de Maistre ou Auguste Comte. Si je garde à l'esprit cette diversité quasi infinie des interprétations et des critiques, alors, en lisant les contributions de nos collègues chinois, je me retrouve très souvent sur un terrain qui m'est familier. Bref, je ne trouve pas ici trace d'une opposition tranchée entre «pensée occidentale» et «pensée chinoise»; j'observe au contraire de multiples rencontres dont j'aimerais dresser un rapide inventaire.

La démocratie occidentale, ai-je dit, est l'ensemble formé par l'État de droit et les institutions représentatives. Cet ensemble est à son tour fondé sur deux principes dont il prétend n'être que la mise en œuvre: la «liberté négative», au sens donné à ce terme par Isaiah Berlin, et la «souveraineté populaire ». Il y a liberté négative lorsque chaque personne est libre de se réaliser comme elle l'entend, le devoir de l'État consistant seulement à lui fournir un cadre dans lequel elle peut agir à son gré selon les valeurs qu'elle s'est elle-même fixées. La liberté ainsi entendue est très proche de la sûreté machiavélienne: elle garantit à chacun un espace inviolable d'autonomie; elle est dite négative parce que tous les contenus positifs du comportement et de l'action sont laissés au libre choix de l'individu.

Quant à la souveraineté populaire, elle signifie d'abord que les décisions qui intéressent la collectivité sont prises par celle-ci ou par ses représentants dûment qualifiés; mais elle implique aussi et surtout que ces décisions sont souveraines : la collectivité statue en dernière instance, ses résolutions ne sont 
pas susceptibles d'appel; il n'existe donc aucune loi, aucune puissance qui viendrait restreindre le pouvoir collectif.

Une première remarque: beaucoup de difficultés que je vais essayer de recenser tiennent au fait - relevé en Occident comme en Chine - que, dans les deux cas, le sujet politique - celui qui exerce la liberté négative et celui qui prend part à la décision collective - est l'individu isolé, abstrait de son environnement social, culturel et historique. En Occident comme en Chine, on s'est d'abord interrogé sur la réalité même d'un être aussi artificiellement produit; comme le dit fort bien Charles Taylor, cité par Lee Ming-huei, «one is self among other selves. A self can never be described without references to those who surround it $»$. En tout état de cause, l'opération qui consiste, dès qu'il s'agit de politique, à séparer l'individu de son contexte et à l'enfermer dans sa solitude n'est en rien innocente; elle est lourde de conséquences quant aux capacités effectivement concédées à l'acteur et quant au sort qui lui est réservé face aux puissances de toutes natures qui l'entourent. Qu'un des hauts lieux de la démocratie ainsi entendue porte le nom d'isoloir en dit davantage sur ce point que bien des commentaires...

Qu'en est-il tout d'abord de la liberté négative ? Comme l'expression même l'indique, elle pose un interdit, mais elle n'énonce aucune prescription. Elle délimite pour chaque individu un espace dans lequel il est protégé de toute interférence extérieure, mais elle ne dit rien sur ce qui peut remplir "positivement» cet espace. À s'en tenir à elle, le risque est donc grand de le voir entièrement ou largement occupé par des intérêts égoïstes et des passions irrationnelles. Par ailleurs, la liberté négative ne connaît pas d'autre limite que la liberté négative d'autrui, mais dès lors que celle-ci est respectée, l'individu n'a pas à se soucier de son prochain; que deviennent dans ces conditions les valeurs de la solidarité, de l'entraide et même de la compassion? Le bénéficiaire de la liberté négative se trouve ainsi déchargé de toute responsabilité à l'égard d'autrui: s'il décide malgré tout de s'en préoccuper, libre à lui, mais rien ne peut l'y obliger. Sous le régime de la liberté négative, «que chacun reste dans son pré, et les troupeaux seront bien gardés ».

En la matière les remarques de Lee Ming-huei et celles de Jiang Qing se rejoignent, et posent les unes comme les autres la question de la référence à une morale. La liberté négative elle-même peut-elle survivre durablement dans la société en l'absence de toute injonction morale ? En vertu de quel impératif les frontières entre ma liberté et celle d'autrui seront-elles respectées? Le raisonnement utilitaire suffira-t-il à garantir ce respect, dès lors que l'inégalité des forces nourrira maintes tentations? En d'autres termes, la société peut-elle entièrement abandonner le sort de la morale à la volonté, mais aussi à l'humeur et au caprice de l'individu ? C'est ici qu'aux yeux de nos collègues la tradition 
confucéenne pourrait apporter à la démocratie un complément indispensable, en particulier du fait du rôle majeur qu'elle accorde à l'éducation.

Quoi qu'il en soit du remède proposé, le souci de Lee Ming-huei et de Jiang Qing est très largement partagé en Occident: venus d'horizons extrêmement divers - depuis l'altermondialisme et l'écologie jusqu' aux Églises et aux communautés religieuses -, d'innombrables procureurs se dressent dans nos pays pour dénoncer le «matérialisme pratique» de la société occidentale, sa soumission aux exigences de la consommation, de l'égoïsme, de la concurrence, du profit et de la volonté de puissance, son manque absolu d'idéal ; ils soulignent à l'envi la désagrégation du lien social que ces vices et carences ne peuvent que nécessairement entraîner. Au nom de l'idéal laïque, et aussi pour se défendre du péril totalitaire, l'Occident a séparé radicalement la politique de la religion, le pouvoir temporel du pouvoir spirituel ; du coup, les confessions religieuses, qui étaient les défenseurs patentés de la loi morale, ont été chassées de l'espace public et reléguées dans la sphère privée. On peut bien entendu s'en réjouir, mais l'amoralisme sans frein que tant de voix dénoncent est de toute évidence la rançon d'une telle transformation. On peut adopter dans ce débat la position que l'on veut, mais on doit bien convenir qu'il n'a rien d'exotique: sous nos yeux, il divise l'Occident comme il divise apparemment la Chine.

En second lieu, la liberté négative est une liberté formelle, en ce sens qu'elle est indifférente aux conditions de sa mise en pratique, donc de son effectivité. Elle ouvre l'espace des choix, mais elle ne nous donne pas les moyens concrets nécessaires à la réalisation de ceux-ci. Tout d'abord, la liberté, même négative, n'est concevable qu'à l'intérieur d'une communauté - aujourd'hui, le plus souvent, d'un État - qui organise son exercice. Mais surtout, elle exige dans la société un minimum d'égalité: si toute une partie de la population est privée des ressources indispensables à sa vie matérielle et spirituelle, la liberté négative dont jouira cette fraction sera dépouillée de tout contenu réel ; la seule possibilité concédée sera de végéter dans la misère. Nous retrouvons ici la célèbre distinction des libertés formelles et des libertés réelles ; l'expérience historique nous a enseigné au prix de beaucoup de sang et de larmes que les unes ne vont pas sans les autres: le combat pour la liberté doit être mené avec la même vigueur sur les deux fronts, et il n'est pas possible - même provisoirement - d'en privilégier un. Dans une société inégalitaire, la bataille pour les seules libertés formelles consolide la formation et le pouvoir d'une élite; mais l'égalité sous la tyrannie n'est que nivellement forcé et asphyxie.

C'est bien dans ce contexte que s'inscrivent les réflexions de Wang Hui. Il souligne l'urgence de «donner la parole à ceux qui sont sans voix »; il montre les pièges dans lesquels nous risquons de tomber à ne défendre que la seule liberté négative: par exemple, la dénonciation de l'emprise excessive de l'État 
peut aussi faire le jeu des grands intérêts privés; de même, à stigmatiser les mouvements sociaux au prétexte qu'ils seraient animés par des utopies aux relents totalitaires, on s'expose aussi à réduire les exclus au silence. La diversité des opinions dans l'espace public - où l'on s'accorde à reconnaître un critère de la démocratie - suppose l'existence de groupes sociaux politisés et mobilisés, faute desquels l'harmonie deviendrait uniformité et conformisme. Bref, nous dit Wang Hui, pas de liberté sans justice. Je n'ai pas besoin de m'étendre beaucoup pour montrer à quel point il y a là une sensibilité qui nous est commune.

J'en viens à la souveraineté populaire: doit-elle être considérée, en régime démocratique, comme la source exclusive du pouvoir? Appartient-elle à l'essence même de la démocratie, et s'il en est bien ainsi, comme on peut en effet le penser, qu'en résulte-t-il pour celle-ci? À nouveau, cette question a été en Occident l'objet de débats interminables. On se rappelle les invectives de Platon contre le régime populaire; celui-ci instaure la tyrannie de la foule; or, la foule n'obéit qu'à ses impulsions affectives et à ses intérêts égoïstes; elle est incapable de raison et de désintéressement. Au surplus, elle est prisonnière de l'instant présent et de la situation immédiate: il lui est impossible de se projeter dans l'avenir; pour la même raison, elle ignore la persévérance et la continuité; elle est susceptible de retournements aussi complets qu'imprévisibles. Comme on sait, cette argumentation sera reprise de siècle en siècle par tous les adversaires aristocratiques de la démocratie, en un concert où, pour m'en tenir aux temps modernes, se mêleront les voix de Montesquieu, de Joseph de Maistre, de Guizot et de Charles Maurras, entre autres. Les démocrates s'efforceront de leur répondre en montrant comment, au sein même de la démocratie, la puissance du peuple peut être contenue, encadrée, domestiquée...

Plus près de nous, une expérience historique cruelle nous a montré que la souveraineté populaire n'est nullement une garantie contre l'autocratie, et Lee Ming-huei a raison de nous rappeler que «tant la démocratie "progressiste" des Jacobins que la démocratie "conservatrice" de Weimar ont donné naissance à des dictatures». Quand des passions comme le fanatisme ou la peur s'emparent du corps social, on ne saurait exclure que celui-ci ne se rue dans la servitude volontaire, même si la souveraineté populaire doit de cette façon creuser sa propre tombe.

Il est donc parfaitement raisonnable de se demander, à l'instar de Jiang Qing, si à côté de la souveraineté populaire, il ne faudrait pas faire place à d'autres sources de légitimité. Dans un exposé très stimulant, Jiang Qing - tout en acceptant l'idée d'une autorité enracinée dans l'adhésion ou la volonté populaire - soutient que deux autres sources devraient être prises en compte pour l'édification d'un ordre social harmonieux et stable. La première est la transcendance ou le sacré, seuls à même de soutenir efficacement les revendications de la morale; 
la seconde est l'histoire et la culture, dont l'individu comme la collectivité sont les produits et les héritiers, et dont ils ne sauraient s'affranchir sans sombrer dans la confusion et se dissoudre dans l'indifférencié.

On peut certainement s'interroger sur ces suggestions, et plus encore sur la traduction institutionnelle qu'en propose Jiang Qing : la création d'un Parlement à trois chambres, dont chacune serait représentative de l'une des légitimités retenues. Mais on ne saurait nier la pertinence de la problématique, et il est de fait qu'en Occident de nombreux États l'ont affrontée dans le passé, et aujourd'hui encore la rencontrent. Je ne parle même pas des démocraties qui, dans leur Constitution même, font référence à une doctrine religieuse, admettant par là que certaines de leurs normes procèdent d'une autre origine que la volonté du peuple. Mais n'en est-il pas de même des États qui, se disant hautement laïques, se réclament cependant d'une «Déclaration universelle des Droits de l'Homme»? Dans ces «Déclarations», les Droits de l'Homme sont posés comme naturels: ils appartiennent à la personne comme telle, de manière innée, avant toute intervention de la société. Celle-ci ne se forme que pour permettre leur réalisation, et le pouvoir n'est légitime que s'il s'engage à les respecter: or, il est clair qu'ils ne doivent rien au suffrage universel et au consentement populaire; au contraire, ils s'imposent à eux et limitent à l'avance le champ où ils peuvent s'exercer.

Les démocraties occidentales elles-mêmes font donc bien appel à une autre légitimité que la souveraineté populaire. Reste à s'interroger sur la nature de cette légitimité, et par conséquent sur le fondement des Droits de l'Homme. Définis comme naturels, ils ne sauraient être le résultat d'une convention dictée par l'utilité; au surplus celle-ci serait bien incapable de leur conférer l'autorité et la dignité qui sont les leurs; on voit donc de nouveau surgir à l'horizon l'éventualité d'une origine transcendante. Par ailleurs, dans la pratique sinon dans les institutions, le rôle de la culture et de l'histoire demeure essentiel dans les démocraties occidentales: aucune d'entre elles ne s'en tient au simple «patriotisme de la constitution»; toutes font référence à un héritage et à une identité, même si celle-ci est perçue comme complexe, mobile et divisée; bien que leur statut soit surtout symbolique, des organismes comme les Académies trouvent ici leur raison d'être. Sur tous ces points, le dialogue avec Jiang Qing peut s'engager de manière fructueuse.

En second lieu, l'équation entre la souveraineté populaire et le système représentatif est rien moins qu'évidente. Un régime qui remet le pouvoir à un corps de mandataires élus et renouvelés à intervalles de plusieurs années est bien davantage une «oligarchie élective» qu'une véritable démocratie. Ici se pose la question de la participation effective du peuple aux débats et aux décisions qui engagent son sort, car, comme l'écrit très bien Wang Hui, «une démocratie 
sans participation n'est qu'une coquille vide, aisément réductible à une pure forme manipulée par telle ou telle force». La réflexion occidentale s'est depuis longtemps saisie de ce problème et elle a examiné ce qui pourrait rapprocher le système représentatif de la démocratie directe, en s'attachant surtout aux procédures juridiques susceptibles de favoriser une association plus étroite du peuple aux «affaires »: recours au référendum, généralisation du mandat impératif, révocabilité des élus, etc. On pourrait élargir l'investigation en se tournant vers d'autres expériences et d'autres cultures : j'ai pour ma part essayé ailleurs de montrer que la palabre africaine offrait un autre mode de délibération et d'action, beaucoup plus attentif à recueillir l'avis de tous, beaucoup plus soucieux également de préserver l'unité du groupe quel que soit l'enjeu de la discussion en cours.

Cependant, l'introduction de nouveaux mécanismes institutionnels ne saurait à elle seule résoudre la difficulté: la véritable solution est de susciter à l'intérieur des masses populaires elles-mêmes, contre toutes les tentations de la résignation et de la modestie, la volonté de prendre effectivement part aux choix collectifs. On ne peut donc ici que se ranger à l'avis de Wang Hui: il s'agit bien de repolitiser la société. En Occident comme en Chine, une intarissable éloquence est répandue pour nous faire croire que désormais la politique a cédé la place à la bonne gouvernance et à l'efficience administrative; en Occident comme en Chine, nous ne maîtriserons notre avenir que dans la mesure où nous saurons mettre cette idéologie en échec.

Un dernier point peut être abordé, en relation avec la question de la participation: à quel(s) niveau(x) la souveraineté populaire doit-elle s'exercer? Je voudrais faire ici mention du remarquable développement que Qin Hui consacre aux rapports entre l'individu d'un côté, les petites et les grandes communautés auxquelles il appartient, de l'autre. En Occident, on l'a vu, et tout particulièrement en France, la liberté négative a été conférée à l'individu isolé, et c'est la collectivité des individus isolés qui forme le peuple souverain. Ont donc été exclues toutes les communautés intermédiaires: les unes - les corporations - ont été purement et simplement abolies; d'autres - la famille, les groupements religieux - ont été rejetées hors du champ politique; d'autres enfin - la commune, le village - n'y ont été admises qu'à un rang subalterne, et leur rôle s'est largement réduit à la transmission et à la mise en œuvre des décisions descendues d'en haut.

Cette exclusion, il faut le souligner, a été présentée comme une opération d'émancipation. Corporations, familles, paroisses, communes ont été décrites comme autant de cellules de confinement et d'oppression, et l'individu s'est vu conseiller de s'appuyer sur l'État afin de mettre un terme aux tutelles dans lesquelles elles le tenaient. Mais sitôt «délivré», il s'est retrouvé seul face à la 
toute-puissance de l'État, et il a bientôt constaté que sa liberté n'y avait guère gagné. La fondation et l'essor du syndicalisme depuis la fin du XIX ${ }^{\mathrm{e}}$ siècle, le formidable élan actuel du mouvement associatif, les efforts tentés pour restituer une certaine marge d'autonomie aux collectivités locales sont autant d'entreprises pour combler le vide qui s'était creusé en 1789 entre l'individu et l'État.

Or, Qin Hui montre très bien comment les réformateurs chinois des premières années du $\mathrm{xx}^{\mathrm{e}}$ siècle se sont trouvés confrontés à un problème de même nature. En présence d'un pouvoir central très affaibli par la chute de l'Empire, la plupart d'entre eux - à la notable exception de Hu Shi - ont estimé que les petites communautés - les lignages, les clans - représentaient le principal foyer de l'oppression, et qu'il fallait donc les prendre pour cibles: ce sont elles qui étaient visées à travers les invectives lancées contre «la boutique confucéenne». Comment cette attitude a favorisé la renaissance, puis la croissance hypertrophique d'un nouveau pouvoir central, c'est, à suivre Qin Hui, un épisode essentiel dans la terrible histoire $\mathrm{du} \mathrm{xx}^{\mathrm{e}}$ siècle chinois.

Les deux expériences conduisent, me semble-t-il, aux mêmes conclusions : la communauté intermédiaire et la communauté supérieure sont l'une et l'autre, comme Janus, des puissances à double face. Chacune d'elles peut être à la fois instrument d'oppression et levier d'émancipation; la seule chance de l'individu, c'est de les jouer l'une contre l'autre, de les neutraliser l'une par l'autre; à lui donc de les maintenir dans un état permanent d'équilibre antagoniste; il pourra ainsi, selon les conjonctures, chercher auprès de l'État une protection contre son clan, ou se réfugier dans son clan pour échapper à l'emprise de l'État. En d'autres termes, qui soutient la cause de la liberté doit s'efforcer de diviser la souveraineté populaire, non seulement, si je puis dire, sur un plan horizontal, en imposant, comme le veut Montesquieu, une stricte séparation des pouvoirs, mais aussi selon un plan vertical, en multipliant les niveaux autonomes de décision et en appliquant de façon rigoureuse le principe de subsidiarité : n'est tranché à l'échelon supérieur que ce qui ne peut pas l'être à l'échelon inférieur.

Je conclus. De la lecture des six textes réunis par Sébastien Billioud et Joël Thoraval, je retire pour ma part un double enseignement. Le premier concerne l'ampleur et la vivacité du débat dans la Chine d'aujourd'hui. Si j'en crois les deux «éditeurs», il ne s'agit nullement ici d'un échantillon qui se prétendrait représentatif; soit, mais à ne considérer que ces six contributions, elles sont à elles seules l'indice de discussions animées, entre des interlocuteurs d'opinions très diverses, parfois même opposées. Mais surtout - second enseignement -, les thèses défendues, les argumentations développées sont remarquablement proches de celles qui circulent au même moment sur la «scène intellectuelle» occidentale et particulièrement française. Plus précisément, j'ai le sentiment d'une sorte d'espace partagé, où chacun s'introduit sans doute avec ses propres références 
historiques et culturelles, mais où se découvrent bientôt d'impressionnantes convergences sur le fond. Certes, des divisions, des contradictions se manifestent de chaque côté de la «frontière», mais compte tenu de la diversité des situations, elles sont de part et d'autre largement congruentes; au-delà des variations liées à la conjoncture, les mêmes «tendances lourdes » sont à l'œuvre ici et là. Faut-il s'en étonner? Après tout, il n'est pas absurde d'imaginer que la mondialisation finisse par produire ses effets jusque dans ce domaine, et qu'un destin de plus en plus commun appelle des réflexions de plus en plus voisines...

Mais peut-être suis-je porté à effacer les différences et à sous-estimer les spécificités? Les spécialistes m'en feront sûrement le reproche. Je ne voudrais pas me montrer ingrat vis-à-vis de ceux d'entre eux qui m'ont accueilli dans cette revue, et dont le travail contribue à la déconstruction de cette illusion d'une différence irréductible. Aussi, c'est à l'un des auteurs cités, Qin Hui, que je demanderai de plaider pour moi. Évoquant la sinologie occidentale au commencement du $\mathrm{xx}^{\mathrm{e}}$ siècle, Qin Hui écrit:

Cette altérité culturelle (ou exotisme) pouvait être admirée ou détestée, mais tous les sinologues s'accordaient à reconnaître le caractère différent ou exotique de la Chine. En effet, sans différence à rechercher, à quoi pouvait bien servir un sinologue? Si tant d'énergie et de ressources sont dépensées pour simplement découvrir que « tous les hommes ont un même esprit et que tous les esprits partagent une même raison (un même principe), à quoi bon une telle entreprise?

Je ne suis pas certain qu' appliquée à notre époque, l'observation de Qin Hui perde du même coup toute sa pertinence. 